tisch diskutierte Themen aus dem rechtlichen Kontext herausgenommen und unter einem anderen Blickwinkel betrachtet, was neue Perspektiven eröffnet und frische Sichtweisen zulässt. Insgesamt handelt es sich bei der Festschrift „Recht, Lehre und Ethik der öffentlichen Verwaltung “ um ein gelungenes Werk und eine empfehlenswerte Lektüre.

\title{
Judith Brockmann/Arne Pilniok, Studieneingangsphase in der Rechtswissenschaft, Nomos 2014
}

Florian Eichel"

Der von Judith Brockmann und Arne Pilniok herausgegebene rechtsdidaktische Band zur „Studieneingangsphase in der Rechtswissenschaft“ entspringt der 4. Jahrestagung des Zentrums für rechtswissenschaftliche Fachdidaktik an der Universität Hamburg im Jahr 2013. Er legt den Fokus auf die eigenen didaktischen Herausforderungen der Studieneingangsphase - definiert als die ersten beiden Studiensemester (S. 12) - und greift damit die Empfehlung des Wissenschaftsrats auf, diesen Studienabschnitt auf neuere Erkenntnisse der hochschuldidaktischen Forschung aufzubauen (S.14 f.). ${ }^{2}$ Nicht nur die Forschung hat neue Erkenntnisse hervorgebracht; auch die Lebensverhältnisse, auf welche die Universitäten reagieren müssen, haben sich geändert (S. 16 f.), und die normativen Zielvorgaben der Länder sind nicht immer auf der Höhe dieser Zeit (S. 35). All das veranschaulicht einen breiten Reformbedarf. Dennoch will der hier zu besprechende Band keine Forderungen für eine Reform der Studienphase formulieren, sondern präsentiert eine fundierte sowie umfangreiche Sammlung verschiedener Konzepte, Denkanstöße und Projekte, die bei aller Vielfalt eine gemeinsame Botschaft in sich tragen: Eine Verbesserung der Lehre in der Studieneingangsphase ist machbar, und lohnt sich Ausprobieren.

Die Lektüre dieses spannenden und wissenschaftlich anspruchsvollen Werkes sei unbedingt empfohlen. Nicht nur Reformwillige an Universitäten und in Ministerien finden hier Anregungen; auch Dozenten, die abseits breit angelegter Reformkonzepte die Gelegenheit suchen, mit Gewinn über die Gestaltung des eigenen Unterrichts nachzudenken, kommen auf ihre Kosten. Es ist deshalb weniger dem Auftrag zu Kritik, als vielmehr dem interessierten Leser geschuldet, vorab die Themen anzusprechen, die er dort nicht finden wird. Das ist zum einen die Frage der Internationalisierung von Rechtswissenschaft und Studium, ${ }^{3}$ die meines Erachtens von der Studieneingangsphase aus konzeptioniert werden muss, aber sicherlich auch Stoff für

* Der Autor ist Privatdozent und Akademischer Rat am Lehrstuhl von Prof. Dr. Wolfgang Hau, Universität Passau.

2 Wissenschaftsrat, Empfehlungen zur Reform des Hochschulzugangs, Drs. 5920/04, S. 60.

3 Kurz berücksichtigt im Beitrag von Krüper, S. 296. 
eine eigene Tagung geben kann, und zum anderen die Frage, welchen Platz andere Wissenschaften (Sozial-, Wirtschafts-, Naturwissenschaften usw.) in der Studieneingangsphase erhalten können und wieviel Freiraum und Antrieb die Studieneingangsphase für solche „Ausflüge “ gewähren muss; ${ }^{4}$ schließlich spielt das e-learning in den einzelnen Beiträgen eine eher geringe Rolle. ${ }^{5}$

Diese Rezension will für die unterschiedlichen Leserinteressen einen Überblick über die Inhalte des Tagungsbandes geben und erlaubt sich daher eine eigene Gliederung. Der Leser findet Beiträge zu den didaktischen Besonderheiten der Studieneingangsphase (I.), Denkanstöße und Konzepte zur institutionellen Verbesserung der Studieneingangsphase (II.) und zur didaktischen Gestaltung des Unterrichts (III.) sowie Einblicke in bereits praktizierte Modelle samt Erfahrungen aus England und der Schweiz (IV.).

\section{Didaktische Besonderheiten der Studieneingangsphase}

Der Beitrag von Lena Stadler und Roland Broemel erforscht, wer die Studierenden von heute sind und auf welche Faktoren die Lehre gerade in der Studieneingangsphase eine Antwort finden muss (S. 37 ff.). Nach einer auf 267 Fragebögen beruhenden, empirisch fundierten Studie der Autoren erweist sich die Aussagekraft der Abiturnote der Studienanfänger für ihren zu erwartenden Erfolg in der Eingangsphase als eher gering (S. 50, $60 \mathrm{ff}$.). Viel entscheidender ist danach die - nicht aus der Abiturnote ablesbare - Sprachkompetenz, die durch Mehrsprachigkeit oder einen Migrationshintergrund beeinflusst sein kann. Dass Sprachkompetenz und Erfolg im Jura-Studium unmittelbar zusammenhängen, ist keine Überraschung; aber dass die herkömmlich praktizierte Didaktik in der Breite darauf noch keine Antwort hat, ruft dieser Beitrag in Erinnerung. Wirklich eine Erkenntnis ist, dass sich die Studierenden verbreitet gar nicht bewusst sind, wie sehr ihre Bildungssprachkompetenz für ihren eigenen Lernerfolg ausschlaggebend ist. Dabei widerstehen die Autoren dem Reflex des Zeitalters der Schlüsselkompetenzkurse und raten richtigerweise davon ab, dem Problem mit begleitenden Kursen zu begegnen; die Lösung sehen sie vielmehr darin, solchen (und ähnlichen) Gegebenheiten bei der Unterrichtsgestaltung und mit Angeboten wie etwa Moot-Courts oder Legal Clinics beizukommen, um der unnötigen Ausgrenzung entgegenzuwirken, die entsteht, wenn solchermaßen behebbare Hürden weder auf Dozenten- noch auf Studentenseite reflektiert werden. Nebenbei vermittelt der Beitrag wertvolle neue Erkenntnisse aus der Empirie, wie sich rechtsdidaktische Studien verlässlich durchführen und damit belastbare Daten für die Forschung gewinnen lassen.

\section{Institutionelle Verbesserung der Studieneingangsphase}

Anja Böning nimmt die im Jahr 1982 erschienene Dissertation von Wolfgang Schütte über „Die Einübung des juristischen Denkens“ zum Ausgangspunkt ihrer Überle-

4 Diesbezüglich immerhin Böning, S. $271 \mathrm{ff}$.

5 Vgl. aber etwa den Beitrag von Lohse/Zwickel, S. 443 ff. 
gungen (S. 260 ff.). Schütte war zu der Erkenntnis gelangt, dass die scheinbar praktische Fokussierung der Ausbildung auf Falllösungen zur Folge habe, dass die juristische Ausbildung von einer Überschätzung dogmatischer Konstrukte geprägt sei und die juristische Beurteilung als ein technischer Vorgang wahrgenommen werde. Dem misst die Autorin unverändert Aktualität bei, um am Ende ihres Beitrags in aller Kürze daraus Lehren für die Umgestaltung der Studieneingangsphase zu ziehen. Bemerkenswert erscheint vor allem der Vorschlag, unter Beteiligung der studentischen Fachschaften einen mobilen Studientag einzurichten, mit dem der Blick auf sozialwissenschaftliche Themen gelenkt werden soll, um zu einem Nachdenken über die ethischen Dimensionen juristischer Berufe anzuregen.

Die Fokussierung des Studiums auf die Fallösung und die damit verbundene Gefahr einer geistigen Monokultur, die den Juristen mehr als technokratischen Rechtsanwender ${ }^{6}$ denn als Motor für die Anpassung und Fortentwicklung des Rechts qualifiziert, greift auch Julian Krüper auf, um ein umfassendes Konzept zum Umbau des ersten Studienjahres zu präsentieren (S. 274 ff.). Nach einem intensiven Plädoyer gegen den Mainstream, Theorie in den Dünkel lebensfremder und nutzloser Wissenschaft zu verweisen, präsentiert Krüper konkrete Gestaltungsvorschläge. Sein Credo ließe sich dabei auf den Slogan zuspitzen: „Grundlagenfächer raus aus dem Grundstudium“. Dass dies überzeugt, liegt weniger daran, dass solchen Fächern im von Anfang an zu examensfixierten Studium der Makel der Nutzlosigkeit angeheftet wird. Vielmehr geraten Grundlagen ohne unmittelbaren Bezug zum Rechtsstoff für den Studienanfänger zu abstrakt, so dass sie in der Tat „an den meisten Studierenden mehr oder weniger wirkungslos vorbeirauschen “. ${ }^{7}$ Den Ausweg aus diesem Dilemma sieht Krüper in der - den gesamten Tagungsband durchziehenden - Erkenntnis, die Grundlagen der Rechtswissenschaft in die Vermittlung des Fachwissens zu integrieren und erst die Schwerpunktphase des Studiums zu nutzen, um Grundlagenfächer in einer eigenen Vorlesung zu präsentieren (Strafrechtsphilosophie im Strafrechtsschwerpunkt, „organisationstheoretisch-rechtssoziologische“ Vorlesung im gesellschaftsrechtlichen Schwerpunkt usw.). Die Integration von Methodik und Rechtstheorie in das Fachwissen lässt sich freilich nur verwirklichen, wenn die Vorlesung „planmäßig“(!) den Anspruch verlieren darf, sie müsse den Stoff komplett abdecken. Hier setzt der zweite Punkt Krüpers an, der an manch einer Universität erprobt wird, mitunter großen Widerstand schafft, aber möglicherweise einfach mal einen Versuch wert ist: Es geht um die bessere Verzahnung und Abstimmung aller von den wissenschaftlichen Mitarbeitern geleiteten Arbeitsgemeinschaften mit der Vorlesung, um Raum für Grundlagenorientierung in der Vorlesung zu gewinnen und einen Teil der Stoffvermittlung mit der fallorientierten Arbeitsgemeinschaft abzudecken (S. $281 \mathrm{f}$. und S. $291 \mathrm{ff}$.).

6 Den vom Autor gewählten Begriff des Rechtsingenieurs möchte ich nicht übernehmen, da mir diese Zunft viel zu patent, ja manchmal als Vorbild für den praktischen Nutzen von Grundlagenorientiertheit erscheint.

7 S. 293; das gilt auch für die Befassung mit ausländischem Recht, S. 296. 
Ziel des Referats von Nora Rzadkowski war es, nach Möglichkeiten zu suchen, die Bildungstheorien und die allgemeine Hochschuldidaktik auch für die rechtswissenschaftliche Fachdidaktik fruchtbar zu machen und sich dabei mit der Empfehlung des Wissenschaftsrats kritisch auseinander zu setzen, Konzepte einer „juristischen Bildung “ zu entwickeln (S. 301 ff.). Ausgangspunkt ihrer Überlegungen ist der Umstand, dass es immer mehr zu wissen gilt und folglich eine strategische Auswahl der Lerngegenstände immer dringender und schwieriger wird. Nach Auffassung der Autorin soll es das Ziel einer rechtswissenschaftlichen Ausbildung sein, Verständigungswissen zu erhalten, das notwendig ist, um sich auszutauschen, während es zu vermeiden gelte, dass das Studium auf die oberflächliche Akkumulation von Wissen hinauslaufe. Als Stütze für eine solchermaßen erforderliche Stoffauswahl wird das wissenschaftliche Modell der „SOLO-Taxonomie“ empfohlen, das den Weg zum Tiefenlernen planbar machen soll.

Der Beitrag von Rolf Schulmeister vom Zentrum für Hochschul- und Weiterbildung der Universität Hamburg liefert die für einen Tagungsband wichtige Außenansicht, geht allerdings leider nicht auf die Besonderheiten der rechtswissenschaftlichen Ausbildung ein (S. 72 ff.). Dennoch ist genau das wiederum die Schlüsselerkenntnis. Dass es gerade im lernintensiven Jura-Studium offenbar keine verlässlichen Erkenntnisse über die Berechnung des Workloads der Studierenden gibt, ist durchaus bemerkenswert. Bei der Gestaltung des rechtswissenschaftlichen Curriculums herrscht daher immer noch eine „Lehrzentrierung anstatt einer Lernzentrierung“ (S. 89), so dass die Auslastung eines Semesters mit Präsenzveranstaltungen immer noch nach Semesterwochenstunden anstatt am studentischen Arbeitsaufwand bemessen wird. Entsprechende Daten zum Arbeitsaufwand wären, wie der Beitrag von Schulmeister belegt, nicht nur für die Gestaltung des Curriculums, sondern auch für dessen Abstimmung mit der Zahl und Gewichtigkeit von Klausuren pro Semester, ihrer zeitlichen Lage und der Gestaltung der vorlesungsfreien Zeit dringend erforderlich. Wer hier methodische Grundlagen sucht, wird in dem für diesen Tagungsband doch etwas überdimensionierten Beitrag (130 S.) mehr als fündig.

\section{Didaktische Gestaltung des Unterrichts}

Tomas Kubn widmet sich der didaktischen Optimierung einer Arbeitsgemeinschaft und wählt als methodischen Ausgangspunkt das Konzept der Fehlerorientierung (S. 470 ff.). Der Beitrag empfiehlt sich auch als Lektüre für Arbeitsgemeinschaftsleiter, da er in Bezug auf zahlreiche Beispiele konkrete und geeignete Handlungsvorschläge entwickelt oder einfach nur zur Reflektion über den eigenen didaktischen Auftrag einlädt. Solche Vorschläge beginnen damit, die den Studierenden gestellten Fragen einmal daraufhin zu durchdenken, ob sie nur Wissen oder aber Begründungen abfragen und dadurch zum Verstehen aktivieren. Zudem wird angeregt, Normen bewusst außerhalb ihres üblichen Zusammenhangs zu thematisieren, um das Verständnis für ihren Wortlaut und ihre Funktionsweise zu schärfen, und es werden Wege beschrieben, um wiederkehrende Wertungen aufzudecken, gesetzliche Begriff- 
lichkeiten zu behandeln, zur Arbeit mit dem Gesetz und zur Gesetzeslektüre anzuhalten und vieles mehr.

Arnd-Christian Kulow unternimmt das Wagnis, psychotherapeutische Ansätze für eine Erstsemesterdidaktik fruchtbar machen zu wollen, nachdem er zunächst die Anforderungen rechtswissenschaftlicher Fachdidaktik unter Rückgriff auf Theorien Niklas Lubmanns bestimmt hat (S. 335 ff.). Die daraus erwachsenden Thesen seiner Untersuchung (beispielsweise Äußerungen über schlechte Berufsaussichten als potentielle Abschreckungsszenarien zu vermeiden, Juristensprache in Alltagssprache zu vermitteln und Erstsemester mit Dozenten zu konfrontieren, die von ihrem Fach begeistert sind) hätten meines Erachtens auch ohne Rückgriff auf die Funktionsweise des zentralen Nervensystems überzeugt, sind dadurch aber immerhin ebenso bunt wie das Fazit Kulows, wonach die juristische Ausbildung „eine Vielzahl von unterschiedlichen und bunten juristischen Vögeln“ schaffe.

Der kurze, aber lebendige Beitrag von Michael Hunter Schwartz und Scott Abbott aus den USA baut auf einer Studie auf, wonach die Person des Rechtsprofessors eine der bedeutendsten Faktoren für den Lernerfolg der Studierenden ist (S. 327 ff.). Unmittelbar einsichtig ist die didaktische Folgerung, Studierende deshalb als Kollegen zu behandeln, ihnen etwas zuzutrauen und zu Fragen aufzufordern, da zu viel Respekt in der Lehrveranstaltung die Hürde für eine breite Beteiligung an Diskussionen zu hoch hängt. Mit Studenten gemeinsam Aufsätze zu veröffentlichen, zu Abend zu essen oder sie nach Hause einzuladen, erscheint dann aber eher auf US-amerikanische Realitäten eines Kleingruppenunterrichts zugeschnitten zu sein.

\section{Einblicke in bereits praktizierte Reformmodelle und Erfahrungen aus anderen Ländern}

Arndt Schmehl stellt den Projektverbund des Universitätskollegs der Universität Hamburg vor (S. 360 ff.). Dieser ist aus der Einsicht gegründet worden, dass die Studieneingangsphase nicht allein durch die Gestaltung des Curriculums oder durch Fachdidaktik verbessert werden kann, sondern einen gezielten und übergreifenden Ansatz benötigt, der neben diesen zwei Faktoren auch die Studienorganisation, die Studierendenbegleitung, die Erwartungen von Lehrenden und Studierendengruppen sowie, allem Widerstand zum Trotz, auch die Phase unmittelbar vor dem Studium einbezieht. Darüber hinaus sollte die institutionelle Verankerung des Projekts in einem Kolleg reformfreudigere Strukturen schaffen. Der Beitrag veranschaulicht die schon erzielten Erfolge des Projekts („Schnupperstudium“ für Schüler; online-basierte Selbsteinschätzungshilfen; Studienbegleitung durch Mentoring) sowie Fragen und Erfahrungen, die es hervorgebracht hat.

Barbara Lange gibt einen ausführlichen Einblick in zwei erfolgreiche Workshops zur Stärkung der Studierkompetenz (S. 376 ff.): Der erste Workshop hat zum Ziel, das Studieren zu lernen, und ist in sechs Module gegliedert (Studierkompetenzen, Lernen im Studium, systematisches Erarbeiten eines Rechtsgebiets, bildhafte Darstellung ju- 
ristischer Inhalte, Zeitmanagement, Lernen durch private Arbeitsgemeinschaften). Im zweiten Workshop geht es um die Verbesserung der Lehre in den Arbeitsgemeinschaften in Form eines - inzwischen auch an verschiedenen Universitäten etablierten - angeleiteten Erfahrungsaustauschs zwischen wissenschaftlichen Mitarbeitern, um deren Bewusstsein für didaktische Möglichkeiten zu schärfen und Raum für neue didaktische Erkenntnisse zu schaffen.

Vanessa Jäger und Julia Speierer stellen das erfolgreiche Regensburger Lern- und Beratungszentrum REGINA vor (Regensburger Individuelles und Nachhaltiges Ausbildungszentrum), ein freiwilliges Zusatzangebot, das Angebote zu Arbeits- und Lernmethoden, zum Klausurenschreibtraining sowie die Möglichkeit zur individuellen Klausurenanalyse enthält, aber auch praxisorientierte Schlüsselqualifikationen vermitteln und Informationen zu juristischen Berufsfeldern und Praktika geben will (S. 408 ff.). Sämtliche Kursformate beruhen inzwischen auf einer Methode, die mit „Wahrnehmen - Austauschen - Üben“ betitelt ist und die Lernende in aktiver Rolle am Lerngeschehen beteiligt. Die Kurse werden von Studierenden an beiden Enden des Leistungsspektrums rege wahrgenommen und sollen in Zukunft auch das „Mittelfeld" verstärkt erreichen.

Eva Julia Lohse und Martin Zwickel liefern einen Werkstattbericht über Angebote zur Schulung juristischer Grundkompetenzen und Arbeitstechniken der Universität Erlangen-Nürnberg (S. 431 ff.). Das Kompetenztraining wird sowohl online als auch durch Präsenzveranstaltungen durchgeführt. Die Inhalte bauen auf den typischen Klausurfehlern auf, vermitteln juristische Kompetenzen wie die Gesetzeslektüre, Methodik der Rechtsanwendung und Argumentationsfähigkeit. Daneben gibt es einen Online-Kurs zur Gutachten- und Klausurtechnik, der inzwischen bayernweit im Rahmen der Virtuellen Hochschule Bayern (VHB) angeboten wird. Hier können die Teilnehmenden auch gegenseitig den Gehalt ihrer Argumentation bewerten.

Mit dem Beitrag von Silvia Pernice-Warnke verlässt der Tagungsband den Fokus auf der Studieneingangsphase, was den Text aber nicht minder interessant macht (S. 454 ff.). Nach einer kurzen Vorstellung des Projekts der Universität zu Köln „Recht aktiv“, durch das Praktika und universitärer Ausbildungsauftrag sinnvoll verzahnt werden, beleuchtet die Untersuchung das didaktische Potential von Praktika für das Studium und wie dieses durch entsprechende Praktikumsbetreuungsprogramme genutzt werden kann.

Christopher Bisping, von der University of Warwick (GB), untersucht das erste Jahr im kurzen Jura-Studium in England und Wales (S. $206 \mathrm{ff}$.), von wo aus das deutsche Studium gerne als „professorenzentriertes System“ kritisch beäugt wird. ${ }^{8}$ Er bietet damit prima facie das Kontrastprogramm zum deutschen Studium, da das Rechtsstudium in England - historisch und institutionell bedingt - ein weniger wissenschaftliches und weit verschulteres als in Deutschland ist und dennoch genauso erfolgreich auf die juristischen Berufe vorbereitet. Interessant sind die berufsständi-

8 Vgl. auch Friedmann, „Übertriebener Status“ in: Der Spiegel 52/2013, S. 34 f. 
schen Vorgaben und die der Akkreditierungsstelle (Quality Assurance Agency for Higher Education), die den Inhalt von Prüfungen und Ausbildung festlegen. Interessant ist auch, dass das, was hierzulande manchmal mit „Softskill“-Kursen als anglo-amerikanische Errungenschaften verkauft wird, in England und Wales gar keine große Rolle spielt. Zur eigenen Verwunderung des Autors hat eine Auswertung der (abgedruckten) Lehrpläne von 20 führenden Law Schools für das erste Jahr ergeben, dass Legal Skills (Recherche der Rechtsquellen, Gesetzeslektüre, Feststellung der Rechtslage, Rechtsanwendung und Auswertung von Forschungsmaterial) in der Regel nicht Gegenstand eigener Kurse sind, sondern einen Schwerpunkt innerhalb der Veranstaltungen zum English Legal System bilden. Hier schließt sich der Kreis zu in anderen Beiträgen dieses Tagungsbands geäußerten Forderungen, in den Vorlesungen mehr Raum für Methodik und andere Grundlagen zu schaffen.

Einblicke in die durch den Bologna-Prozess erheblich umgestaltete Eingangsphase des juristischen Studiums in der Schweiz verschafft schließlich der Beitrag von Andreas Thier, Gwendolin Bodemann und Alessia Dedual von der Universität Zürich (S. $229 \mathrm{ff}$.). Die Umgestaltung in ein dreistufiges Studium, bestehend aus Bachelor, Master und Doktorat, sowie in ein Kreditpunktesystem hat den ersten zwei Semestern eine sehr rigide Auswahlfunktion verliehen, so dass inzwischen die Feststellung der Studienbefähigung im Mittelpunkt der schweizerischen Studieneingangsphase steht. Dadurch gerät die gleichfalls mit der Reform verbundene Zielsetzung, nicht allein Fachkenntnisse, sondern ein juristisches Grundverständnis zu vermitteln, unter Druck. Der „Weg vom Inhalt zur Methode“ (S. 252) stellt die juristische Hochschullehre in der Schweiz eben auch deshalb vor neue Herausforderungen, da die Auswahlfunktion und der damit verbundene Prüfungsdruck und -fokus den für die Verwirklichung dieser Ziele notwendigen Faktoren entgegenwirken. Auch das läuft, wie explizit schon bei den Beiträgen von Stadler und Broemel, von Krüper und von Bisping - nur eben aus anderem Blickwinkel - wieder auf eine Betonung der Notwendigkeit hinaus, die Methode zu einem integralen Bestandteil der Wissensvermittlung zu machen.

Das eignet sich denn auch als ein Fazit zu diesem facettenreichen, inspirierenden und auch wissenschaftlich fundierten Tagungsband, dessen Lektüre ein erhellendes didaktisches Erlebnis gewesen ist. 